Proceedings of the Institute of Mathematics and Mechanics,

National Academy of Sciences of Azerbaijan

Volume 45, Number 2, 2019, Pages 213-221

https://doi.org/10.29228/proc.5

\title{
ON EIGENVALUES OF A BOUNDARY VALUE PROBLEM FOR A SECOND ORDER ELLIPTIC DIFFERENTIAL-OPERATOR EQUATION
}

\author{
BAHRAM A. ALIEV
}

\begin{abstract}
In a separable Hilbert space $H$, we study the asymptotic behavior of eigenvalues of a boundary value problem for a second order elliptic differential-operator equation in the case when one, and the same, spectral parameter participates linearly in the equation and quadratically in the boundary conditions. Asymptotic formulas are obtained for eigenvalues of the considered boundary value problems, one of the series converges to zero.
\end{abstract}

\section{Introduction}

In this paper, in a separable Hilbert space $H$, we study the asymptotic behavior of the eigenvalues of the following boundary value problem for a second order elliptic differential -operator equation

$$
\begin{gathered}
-u^{\prime \prime}(x)+A u(x)=\lambda u(x), x \in(0,1), \\
u^{\prime}(0)+\lambda^{2} u(0)=0, \\
u^{\prime}(1)-\lambda^{2} u(1)=0,
\end{gathered}
$$

where $\lambda$ is a spectral parameter, $A$ is a self-adjoint, positive-definite operator in $H$ and the inverse operator $A^{-1}$ is completely continuous in $H$. It is proved that the eigenvalues of the boundary value problem (1.1), (1.2) are real and simple. Then, it is shown that problem (1.1), (1.2) has three series of eigenvalues, one of which converges to zero, and the other two ones asymptotically behave as $(2 n)^{2} \pi^{2}+\mu_{k}$ and $(2 n-1)^{2} \pi^{2}+\mu_{k}$, where $\mu_{k}=\mu_{k}(A)$ are the eigenvalues of the operator $A$.

Asymptotic behavior of the eigenvalues of boundary value problems for the equation (1.1) with the boundary conditions

$$
u^{\prime}(0)+\lambda u(0)=0, u(1)=0,
$$

was studied in the papers [8], [12], while with boundary conditions

$$
u^{\prime}(0)+\lambda u(0)=0, u^{\prime}(1)-\lambda u(1)=0,
$$

2010 Mathematics Subject Classification. 35J25, 35P20.

Key words and phrases. differential-operator equation, spectral parameter in boundary conditions, eigenvalue, asymptotic formula. 
in [1], where it was proved that a set of the eigenvalues of boundary value problems (1.1), (1.3) and (1.1), (1.4) is discrete and has two series of eigenvalues: $\lambda_{k} \sim \sqrt{\mu_{k}}$, $\lambda_{k, n} \sim \mu_{k}+n^{2} \pi^{2} . k, n \in N$.

The similar issue was studied in [2] for equation (1.1) with boundary conditions of the form

$$
u^{\prime}(0)+d \lambda^{2} u(0)=0, u(1)=0,
$$

where $d$ is some positive number. Peculiarity of boundary value problem (1.1), (1.5) is that this problem has two series of eigenvalues, one which converges to zero, i.e., for boundary value problem (1.1), (1.5) the classical case is violated.

In [13], it is shown that for the squared Laplace equation, there exists a spectral problem with a nonclassical asymptotics, i.e., the problem under consideration, in which one of the boundary conditions contains a differential operator, has a sequence of eigenvalues converging to zero.

The asymptotic behavior of the eigenvalues of boundary value problems for second order differential-operator equation when, unlike the papers [8], [12], [1],[2], one, and the same, parameter enters quadratically into the equation and linearly into the boundary conditions, was studied in [3], [4], [5].

In $[6],[7]$, in a Hilbert space, the solvability of boundary value problems was studied for elliptic differential- operator equations with a spectral parameter in the equation and in the boundary conditions. Note that, in these papers, the boundary conditions, in addition to a spectral parameter, contain linear operators as well.

Boundary value problems with a spectral parameter in the equation and in the boundary conditions for second order ordinary differential equations, in contrast to boundary value problems for second order elliptic differential-operator equations, have been studied in different aspects and deeper. Note some of them.

In [9], [10], a boundary value problem for a second order ordinary differential equation is considered in the case when one, and the same, spectral parameter $\lambda$ linearly participates in the equation and quadratically in one of the boundary conditions. In particular, the asymptotic behavior of the eigenvalues of the considered boundary value problems was studied.

In [11], a boundary value problem for a second order ordinary differential equation is considered when one, and the same, spectral parameter $\lambda$ quadratically participates in the equation, while in the boundary conditions it appears as a quadratic trinomial (with respect to $\lambda$ ). In particular, the asymptotic behavior of eigenvalues of the considered boundary value problems is studied.

In [14], a spectral problem for ordinary differential equations with a quadratic spectral parameter in the equation and in the boundary conditions is considered, where it is proved that the spectrum of the considered boundary value problems is discrete and the system of root functions is two fold complete in certain spaces.

\section{Some properties of eigenvalues}

Lemma 2.1. The eigenvalues of boundary value problem (1.1), (1.2) are real.

Proof. Denote the eigenvectors of the operator $A$, that correspond to the eigenvalues $\mu_{k}$, by $\varphi_{k}, k \in N$. It is known, that the system $\left\{\varphi_{k}\right\}$ forms a complete orthonormal basis in the space $H$. Then, from the expansion $u(x)=$ 
$\sum_{k=1}^{\infty}\left(u(x), \varphi_{k}\right)_{H} \varphi_{k}$, for the Fourier coefficients $u_{k}(x)=\left(u(x), \varphi_{k}\right)_{H}$, we get the following spectral problem:

$$
\begin{gathered}
-u_{k}^{\prime \prime}(x)+\mu_{k} u_{k}(x)=\lambda u_{k}(x), \quad x \in(0,1), \\
u_{k}^{\prime}(0)+\lambda^{2} u_{k}(0)=0 \\
u_{k}^{\prime}(1)-\lambda^{2} u_{k}(1)=0
\end{gathered}
$$

Thus, the study of eigenvalues of boundary value problem (1.1), (1.2) is reduced to the study of eigenvalues of boundary value problem $(2.1),(2.2)$, for various natural $k$. The spectrum of boundary value problem (1.1), (1.2) consists of those $\lambda$ for which problem $(2.1),(2.2)$ has a nontrivial solution $u_{k}(x)$, at least for one $k$. The number $\lambda=\mu_{k}$ cannot be an eigenvalue of problem (2.1), (2.2), as in this case this problem has only a trivial solution.

Let $\lambda$ be an eigenvalue of boundary value problem $(2.1),(2.2)$ and $u_{k}(x, \lambda)$ be the corresponding eigenfunction. Multiplying the both sides of equality (2.1) by the function $\overline{u_{k}(x, \lambda)}$ and integrating the obtained identity with respect to $x$ from 0 to 1 , we have:

$$
-\int_{0}^{1} u_{k}^{\prime \prime}(x, \lambda) \overline{u_{k}(x, \lambda)} d x+\mu_{k} \int_{0}^{1}\left|u_{k}(x, \lambda)\right|^{2} d x=\lambda \int_{0}^{1}\left|u_{k}(x, \lambda)\right|^{2} d x .
$$

Calculating the frist integral by parts and using boundary conditions (2.2), we get:

$$
\begin{aligned}
& \int_{0}^{1} u_{k}^{\prime \prime}(x, \lambda) \overline{u_{k}(x, \lambda)} d x=\left.\overline{u_{k}(x, \lambda)} u_{k}^{\prime}(x, \lambda)\right|_{0} ^{1}-\int_{0}^{1} u_{k}^{\prime}(x, \lambda) \overline{u_{k}^{\prime}(x, \lambda)} d x= \\
& =\overline{u_{k}(1, \lambda)} u_{k}^{\prime}(1, \lambda)-\overline{u_{k}(0, \lambda)} u_{k}^{\prime}(0, \lambda)-\int_{0}^{1}\left|u_{k}^{\prime}(x, \lambda)\right|^{2} d x= \\
& =\lambda^{2} \overline{u_{k}(1, \lambda)} u_{k}(1, \lambda)+\lambda^{2} \overline{u_{k}(0, \lambda)} u_{k}(0, \lambda)-\int_{0}^{1}\left|u_{k}^{\prime}(x, \lambda)\right|^{2} d x= \\
& =\lambda^{2}\left(\left|u_{k}(1, \lambda)\right|^{2}+\left|u_{k}(0, \lambda)\right|^{2}\right)-\int_{0}^{1}\left|u_{k}^{\prime}(x, \lambda)\right|^{2} d x .
\end{aligned}
$$

Hence, from (2.3), it follows:

$$
\begin{aligned}
& \lambda^{2}\left(\left|u_{k}(0, \lambda)\right|^{2}+\left|u_{k}(1, \lambda)\right|^{2}\right)+\lambda \int_{0}^{1}\left|u_{k}(x, \lambda)\right|^{2} d x- \\
& -\int_{0}^{1}\left|u_{k}^{\prime}(x, \lambda)\right|^{2} d x-\mu_{k} \int_{0}^{1}\left|u_{k}(x, \lambda)\right|^{2} d x=0 .
\end{aligned}
$$


Denote:

$$
\begin{aligned}
& a_{k}(\lambda)=\left|u_{k}(0, \lambda)\right|^{2}+\left|u_{k}(1, \lambda)\right|^{2}, \quad b_{k}(\lambda)=\int_{0}^{1}\left|u_{k}(x, \lambda)\right|^{2} d x, \\
& c_{k}(\lambda)=-\int_{0}^{1}\left|u_{k}^{\prime}(x, \lambda)\right|^{2} d x-\mu_{k} \int_{0}^{1}\left|u_{k}(x, \lambda)\right|^{2} d x .
\end{aligned}
$$

In these notation, from (2.5), it follows that the eigenvalue $\lambda$ is a root of the equation

$$
a_{k}(\lambda) z^{2}+b_{k}(\lambda) z+c_{k}(\lambda)=0
$$

for every $k$. As $a_{k}(\lambda) \geq 0, b_{k}(\lambda)>0, c_{k}(\lambda)<0$, for any $k \in N$, then $b_{k}^{2}(\lambda)-$ $4 a_{k}(\lambda) c_{k}(\lambda)>0$. Consequently, for every $k$, the problem $(2.1),(2.2)$ has only real $\lambda$ roots. Lemma 2.1 is proved.

Lemma 2.2. The eigenvalues of boundary value problem (1.1), (1.2) are prime.

As was noted, the problem (1.1), (1.2) is reduced to the study of spectral problem $(2.1),(2.2)$, for every $k$. The similar lemma for boundary value problems of the form (2.1), (2.2) was proved in [11]. Therefore we omit the proof of Lemma 2.2.

Lemma 2.3. The number $\lambda=0$ is not an eigenvalue of the boundary value problem (1.1), (1.2).

Proof. It is sufficient to prove that boundary value problem (2.1), (2.2), for $\lambda=0$, i.e., the problem

$$
\begin{gathered}
-u_{k}^{\prime \prime}(x)+\mu_{k} u_{k}(x)=0, \quad x \in(0,1), \\
u_{k}^{\prime}(0)=0, u_{k}^{\prime}(1)=0,
\end{gathered}
$$

for every natural $k$, has only a trivial solution. The general solution of equation (2.7) has the form

$$
u_{k}(x)=c_{1} e^{-x \sqrt{\mu_{k}}}+c_{2} e^{-(1-x) \sqrt{\mu_{k}}},
$$

where $c_{i}, i=1,2$ are arbitrary constants. Taking (2.9) into account in (2.8), we get a system with respect to $c_{i}$, whose determinant has the form

$$
D=\mu_{k}\left(e^{-2 \sqrt{\mu_{k}}}-1\right) \text {. }
$$

Obviously, for every $k, D \neq 0$. Hence, it follows that for any $k$, the function $u_{k}(x)$, determined by formula $(2.9)$, is identically equal to zero, i.e., $\lambda=0$ is not an eigenvalue of boundary value problem (2.1), (2.2), or of boundary value problem (1.1), (1.2). Lemma 2.3 is proved.

\section{Asymptotic formulas for eigenvalues}

Theorem 3.1. Let $A$ be a self-adjoint, positive-definite operator in a separable Hilbert space $H$ and $A^{-1}$ be completely continuous in $H$.

Then boundary value problem (1.1), (1.2) has three series of eigenvalues: $\lambda_{k}^{(1)} \rightarrow$ 0 as $k \rightarrow \infty$ and $\lambda_{k, n}^{(2)}=\mu_{k}+\gamma_{n}, \lambda_{k, n}^{(3)}=\mu_{k}+\delta_{n}, k, n \in N$, where $\mu_{k}=\mu_{k}(A) \rightarrow$ $+\infty$ are the eigenvalues of the operator $A, \gamma_{n} \sim(2 n-1)^{2} \pi^{2}, \delta_{n} \sim(2 n)^{2} \pi^{2}$ as $n \rightarrow \infty$. 
Proof. The general solution of ordinary differential equation (2.1) is of the form

$$
u_{k}(x, \lambda)=c_{1} e^{-x \sqrt{\mu_{k}-\lambda}}+c_{2} e^{-(1-x) \sqrt{\mu_{k}-\lambda}},
$$

where $c_{i}, i=1,2$ are arbitrary constants. Taking (3.1) into account in boundary conditions (2.2), we get a system with respects to $c_{i}, i=1,2$, whose determinant has the form

$$
D(\lambda)=\left(\lambda^{2}+\sqrt{\mu_{k}-\lambda}\right)^{2} e^{-2 \sqrt{\mu_{k}-\lambda}}-\left(\lambda^{2}-\sqrt{\mu_{k}-\lambda}\right)^{2} .
$$

Thus, the eigenvalues of boundary value problem (2.1), (2.2) and, therefore, of boundary value problem (1.1), (1.2), are zeros of the equation $D(\lambda)=0$ (with respect to $\left.\lambda, \lambda \neq \mu_{k}\right)$ at least for one $k$ :

$$
\left(\lambda^{2}+\sqrt{\mu_{k}-\lambda}\right)^{2} e^{-2 \sqrt{\mu_{k}-\lambda}}-\left(\lambda^{2}-\sqrt{\mu_{k}-\lambda}\right)^{2}=0 .
$$

Write equation (3.2) in the form of a system of equations

$$
\begin{aligned}
& \left(\lambda^{2}+\sqrt{\mu_{k}-\lambda}\right) e^{-\sqrt{\mu_{k}-\lambda}}+\left(\lambda^{2}-\sqrt{\mu_{k}-\lambda}\right)=0, \\
& \left(\lambda^{2}+\sqrt{\mu_{k}-\lambda}\right) e^{-\sqrt{\mu_{k}-\lambda}}-\left(\lambda^{2}-\sqrt{\mu_{k}-\lambda}\right)=0 .
\end{aligned}
$$

Thus, the eigenvalues of the boundary value problem (2.1), (2.2) consist of those real $\lambda \neq \mu_{k}$, that at least for one $k$, satisfy at least one of the equations, (3.3) or (3.4).

Rewrite the system (3.3), (3.4) in the form.

$$
\begin{aligned}
& \lambda^{2} \operatorname{ch}\left(\frac{1}{2} \sqrt{\mu_{k}-\lambda}\right)-\sqrt{\mu_{k}-\lambda} \operatorname{sh}\left(\frac{1}{2} \sqrt{\mu_{k}-\lambda}\right)=0, \\
& \lambda^{2} \operatorname{sh}\left(\frac{1}{2} \sqrt{\mu_{k}-\lambda}\right)-\sqrt{\mu_{k}-\lambda} \operatorname{ch}\left(\frac{1}{2} \sqrt{\mu_{k}-\lambda}\right)=0 .
\end{aligned}
$$

First, study equation (3.5). Find those eigenvalues $\lambda$, for which $\lambda<\mu_{k}$. We will consider the cases $\lambda<0$ and $0<\lambda<\mu_{k}$ separately. Assume $\sqrt{\mu_{k}-\lambda}=y$. Hence $\lambda=\mu_{k}-y^{2}, \lambda^{2}=\left(\mu_{k}-y^{2}\right)^{2}$. If $\lambda<0$ then $\sqrt{\mu_{k}}<y<+\infty$. Then equation (3.5) takes the form

$$
\left(\mu_{k}-y^{2}\right)^{2} \operatorname{ch}\left(\frac{y}{2}\right)-y \operatorname{sh}\left(\frac{y}{2}\right)=0, \sqrt{\mu_{k}}<y<+\infty .
$$

Equation (3.7) is equivalent to the equation

$$
\left(\mu_{k}-y^{2}\right)^{2}-y \operatorname{th}\left(\frac{y}{2}\right)=0, \sqrt{\mu_{k}}<y<+\infty .
$$

Equation (3.8) is equivalent to the equation

$$
\left(y^{2}-\mu_{k}\right)-\sqrt{y t h\left(\frac{y}{2}\right)}=0, \quad \sqrt{\mu_{k}}<y<+\infty .
$$

Let us consider the function $f_{k}(y)=\left(y^{2}-\mu_{k}\right)-\sqrt{y \operatorname{th}\left(\frac{y}{2}\right)}, y \in\left(\sqrt{\mu_{k}},+\infty\right)$. The derivative

$$
f_{k}^{\prime}(y)=2 y-\frac{1}{2 \sqrt{y \operatorname{th}\left(\frac{y}{2}\right)}} \cdot \frac{\operatorname{sh} y+y}{2 \operatorname{ch}^{2}\left(\frac{y}{2}\right)}=\frac{4 \sqrt{2} y \sqrt{y \operatorname{shy}} \operatorname{ch}\left(\frac{y}{2}\right)-\operatorname{shy}-y}{2 \sqrt{2} \sqrt{y \operatorname{shy}} \operatorname{ch}\left(\frac{y}{2}\right)},
$$


for $y \in\left(\sqrt{\mu_{k}},+\infty\right)$. Obviously, for any $y>\sqrt{\mu_{k}}$,

$$
4 \sqrt{2} y \sqrt{y \operatorname{sh} y} \operatorname{ch}\left(\frac{y}{2}\right)-\operatorname{sh} y-y>0 .
$$

Indeed, for any $y>\sqrt{\mu_{k}}$,

$$
\begin{gathered}
4 \sqrt{2} y \sqrt{y \operatorname{shy}} \operatorname{ch}\left(\frac{y}{2}\right)-\operatorname{sh} y-y=\operatorname{sh} y\left[4 \sqrt{2} y \sqrt{\frac{y}{\operatorname{sh} y}} \operatorname{ch}\left(\frac{y}{2}\right)-1\right]-y= \\
=\operatorname{sh} y\left[4 y^{3 / 2} \sqrt{\operatorname{cth}\left(\frac{y}{2}\right)}-1\right]-y>\operatorname{sh} y\left[4 y^{3 / 2} \sqrt{\operatorname{cth}\left(\frac{y}{2}\right)}-1\right]-\operatorname{sh} y= \\
=2 \operatorname{sh} y\left[2 y^{3 / 2} \sqrt{\operatorname{cth}\left(\frac{y}{2}\right)}-1\right] .
\end{gathered}
$$

Without loss of generality we suppose that for any $k, \mu_{k} \geq 1$. It is clear if $y \geq 1$, then the inequality $\operatorname{cth}\left(\frac{y}{2}\right)>\frac{1}{4 y 3}$ is true. From here we have if $y \geq 1$, it follows that $2 y^{\frac{3}{2}} \sqrt{\operatorname{cth}\left(\frac{y}{2}\right)}-1>0$. This, if show that if, $y \in\left(\sqrt{\mu_{k}},+\infty\right)$ then $f_{k}^{\prime}(y)>0$.

So, $f_{k}(y)$ strongly monotonically increases on $\left(\sqrt{\mu_{k}},+\infty\right)$ for every $k$.

Taking into account that, for any $k$,

$$
\begin{gathered}
f_{k}\left(\sqrt{\mu_{k}}\right)=\lim _{y \rightarrow \sqrt{\mu_{k}}} f_{k}(y)=\lim _{y \rightarrow \sqrt{\mu_{k}}}\left(\left(y^{2}-\mu_{k}\right)-\sqrt{y t h\left(\frac{y}{2}\right)}\right)= \\
=-\sqrt{\sqrt{\mu_{k}} \operatorname{th}\left(\frac{\sqrt{\mu_{k}}}{2}\right)}<0 .
\end{gathered}
$$

and

$$
\begin{aligned}
f_{k}(+\infty)= & \lim _{y \rightarrow+\infty} f_{k}(y)=\lim _{y \rightarrow+\infty}\left[\left(y^{2}-\mu_{k}\right)-\sqrt{y \operatorname{th}\left(\frac{y}{2}\right)}\right]= \\
& =\lim _{y \rightarrow+\infty} \frac{\left(y^{2}-\mu_{k}\right)^{2}-y \operatorname{th}\left(\frac{y}{2}\right)}{\left(y^{2}-\mu_{k}\right)+\sqrt{y \operatorname{th}\left(\frac{y}{2}\right)}}=+\infty,
\end{aligned}
$$

we conclude, that in the interval $\left(\sqrt{\mu_{k}},+\infty\right)$, the function $f_{k}(y)$ has exactly one zero $y_{k}$. Show that the zeros $y_{k}$, as $k \rightarrow \infty$, asymptotically behave as $\sqrt{\mu_{k}}$. Indeed, for sufficiently small $\varepsilon>0$, the relation

$$
\begin{gathered}
\frac{f_{k}\left(\sqrt{\mu_{k}}+\varepsilon\right)}{\sqrt{\mu_{k}}+\varepsilon}=\frac{\left(\sqrt{\mu_{k}}+\varepsilon\right)^{2}-\mu_{k}-\sqrt{\left(\sqrt{\mu_{k}}+\varepsilon\right) \operatorname{th}\left(\frac{\sqrt{\mu_{k}}+\varepsilon}{2}\right)}}{\sqrt{\mu_{k}}+\varepsilon}= \\
=\frac{2 \varepsilon \sqrt{\mu_{k}}+\varepsilon^{2}}{\sqrt{\mu_{k}}+\varepsilon}-\sqrt{\frac{1}{\sqrt{\mu_{k}}+\varepsilon} \operatorname{th}\left(\frac{\sqrt{\mu_{k}}+\varepsilon}{2}\right)},
\end{gathered}
$$

as $k \rightarrow+\infty$, tends to $2 \varepsilon>0$. Consequently, beginning with some $k$, the value $f_{k}\left(\sqrt{\mu_{k}}+\varepsilon\right)>0$. On the other hand, for any $k, f_{k}\left(\sqrt{\mu_{k}}\right)<0$. Thus, $y_{k}$ lies between $\sqrt{\mu_{k}}$ and $\sqrt{\mu_{k}}+\varepsilon$. By arbitrariness of $\varepsilon$, we have the equivalence $y_{k} \sim \sqrt{\mu_{k}}$, as $k \rightarrow \infty$. Hence, from the equality $\sqrt{\mu_{k}-\lambda}=y$, for the eigenvalues of the boundary value problem (2.1), (2.2), satisfying the condition $\lambda<0$, we get the following asymptotic relation: $\lambda_{k}^{(1)} \rightarrow 0$, as $k \rightarrow \infty$. 
Now, let as consider the case when $0<\lambda<\mu_{k}$. In this case, equation (3.5) is studied in the same way as in the case of $\lambda<0$. After substitution of $\sqrt{\mu_{k}-\lambda}=y$ $\left(0<y<\sqrt{\mu_{k}}\right)$, equation (3.5) takes the from

$$
\left(\mu_{k}-y^{2}\right)^{2} \operatorname{ch}\left(\frac{y}{2}\right)-y \operatorname{sh}\left(\frac{y}{2}\right)=0, \quad 0<y<\sqrt{\mu_{k}},
$$

or

$$
\left(\mu_{k}-y^{2}\right)-\sqrt{y t h\left(\frac{y}{2}\right)}=0,0<y<\sqrt{\mu_{k}} .
$$

Let us consider the function $\psi_{k}^{\prime}(y)=\left(\mu_{k}-y^{2}\right)-\sqrt{y t h\left(\frac{y}{2}\right)}, y \in\left(0, \sqrt{\mu_{k}}\right)$. Then the derivative

$$
\psi_{k}^{\prime}(y)=-2 y-\frac{1}{2 \sqrt{y \operatorname{th}\left(\frac{y}{2}\right)}} \cdot \frac{\operatorname{sh} y+y}{2 \operatorname{ch}^{2}\left(\frac{y}{2}\right)}<0
$$

for $y \in\left(0, \sqrt{\mu_{k}}\right)$. So, $\psi_{k}(y)$ strictly monotonically decreases on $\left(0, \sqrt{\mu_{k}}\right)$, for every $k$. Taking into account that, for any $k$,

$$
\psi_{k}(0)=\lim _{y \rightarrow 0+} \psi_{k}(y)=\lim _{y \rightarrow 0+}\left(\left(\mu_{k}-y^{2}\right)-\sqrt{y t h\left(\frac{y}{2}\right)}\right)=\mu_{k}>0
$$

and

$$
\psi_{k}\left(\sqrt{\mu_{k}}\right)=\lim _{y \rightarrow \sqrt{\mu_{k}}} \psi_{k}(y)=-\sqrt{\sqrt{\mu_{k}} t h\left(\frac{\sqrt{\mu_{k}}}{2}\right)}<0,
$$

we conclude, that in the interval $\left(0, \sqrt{\mu_{k}}\right)$, the function $\psi_{k}(y)$ has exactly one zero $y_{k}$. Show that the zeros $y_{k}$, as $k \rightarrow \infty$, asymptotically behave as $\sqrt{\mu_{k}}$. Indeed, for sufficiently small $\varepsilon>0$, the relation

$$
\begin{gathered}
\frac{\psi_{k}\left(\sqrt{\mu_{k}}-\varepsilon\right)}{\sqrt{\mu_{k}}-\varepsilon}=\frac{\mu_{k}-\left(\sqrt{\mu_{k}}-\varepsilon\right)^{2}-\sqrt{\left(\sqrt{\mu_{k}}-\varepsilon\right) \operatorname{th}\left(\frac{\sqrt{\mu_{k}}-\varepsilon}{2}\right)}}{\sqrt{\mu_{k}}-\varepsilon}= \\
=\frac{2 \varepsilon \sqrt{\mu_{k}}-\varepsilon^{2}}{\sqrt{\mu_{k}}-\varepsilon}-\sqrt{\frac{1}{\sqrt{\mu_{k}}-\varepsilon} \operatorname{th}\left(\frac{\sqrt{\mu_{k}}-\varepsilon}{2}\right)},
\end{gathered}
$$

as $k \rightarrow \infty$, tends to $2 \varepsilon>0$. Consequently, beginning with some $k$, the value $\psi_{k}\left(\sqrt{\mu_{k}}-\varepsilon\right)>0$. On the other hand, for any $k$, as shown above, $\psi_{k}\left(\sqrt{\mu_{k}}\right)<0$.

Thus, $y_{k}$ lies between $\sqrt{\mu_{k}}-\varepsilon$ and $\sqrt{\mu_{k}}$, starting with some $k$. By arbitrariness of $\varepsilon$ we have the equivalence $y_{k} \sim \sqrt{\mu_{k}}$ as $k \rightarrow \infty$. Hence, from the equality $\sqrt{\mu_{k}-\lambda}=y$, for the eigenvalues of the boundary value problem (2.1),(2.2), satisfying the condition $0<\lambda<\mu_{k}$, we get the following asymptotic relation: $\lambda_{k}^{(1)} \rightarrow 0$ as $k \rightarrow \infty$. Thus, for $-\infty<\lambda<\mu_{k}$, problem (2.1),(2.2) has one series of eigenvalues convergent to zero.

Now, we find those eigenvalues $\lambda$ for which $\lambda>\mu_{k}$. Substitute in equation (3.5) $\sqrt{\lambda-\mu_{k}}=z \quad(0<z<+\infty)$. Then it takes the form

$$
\left(z^{2}+\mu_{k}\right)^{2} \cos \left(\frac{z}{2}\right)+z \sin \left(\frac{z}{2}\right)=0, \quad z \in(0,+\infty) .
$$

Let $z \neq(2 n-1) \pi, n \in N$. In this case, equation (3.10) is equivalent to the equation

$$
\left(z^{2}+\mu_{k}\right)^{2}+z \operatorname{tg}\left(\frac{z}{2}\right)=0, \quad z \in(0,+\infty), z \neq(2 n-1) \pi, \quad n \in N .
$$


Let us consider the function

$$
\varphi_{k}(z)=\left(z^{2}+\mu_{k}\right)^{2}+z t g\left(\frac{z}{2}\right), \quad z \in(0,+\infty), z \neq(2 n-1) \pi, \quad n \in N
$$

Since in each interval $((2 n-1) \pi,(2 n+1) \pi), \quad n \in N$, the function $\varphi_{k}(z)$ takes the values from $-\infty$ to $+\infty$, and its derivative

$$
\varphi_{k}^{\prime}(z)=4 z\left(z^{2}+\mu_{k}\right)+\frac{\sin z+z}{2 \cos ^{2}\left(\frac{z}{2}\right)}>0,
$$

then, in it, for every $k$, the function $\varphi_{k}(z)$ has only one zero $z_{k, n}$ : $(2 n-1) \pi<$ $z_{k, n}<(2 n+1) \pi, \quad n \in N$. Find, for every $k \in N$, asymptotic formulas for $z_{k, n}$, as $n \rightarrow \infty$. From (3.11), we have

$$
\operatorname{tg}\left(\frac{z}{2}\right)=-\frac{\left(\mu_{k}+z^{2}\right)^{2}}{z}, \quad z \in(0,+\infty), z \neq(2 n-1) \pi, \quad n \in N .
$$

Denote $q_{k}(z)=-\frac{\left(\mu_{k}+z^{2}\right)^{2}}{z}, \quad z \in(0,+\infty)$. Obviously, for every $k, q_{k}(z)<0$ and $q_{k}^{\prime}(z)=-\frac{\left(\mu_{k}+z^{2}\right)\left(3 z^{2}-\mu_{k}\right)}{z^{2}}, q_{k}^{\prime \prime}(z)=-\frac{6 z^{4}+2 \mu_{k}^{2}}{z^{3}}<0$. The points $z_{k}=\sqrt{\frac{\mu_{k}}{3}}$ are critical points of the function $q_{k}(z)$. If $z_{k}>\sqrt{\frac{\mu_{k}}{3}}$ then, $q_{k}^{\prime}(z)<0$; if $0<z_{k}<\sqrt{\frac{\mu_{k}}{3}}$, then $q_{k}^{\prime}(z)>0$, i.e., the function $q_{k}(z)$ increases in the interval $\left(0, \sqrt{\frac{\mu_{k}}{3}}\right)$ and decreases in the interval $\left(\sqrt{\frac{\mu_{k}}{3}},+\infty\right)$, for every $k$. So, $q_{k}(z)$ is a negative, strictly upward convex function, for every $k$. Moreover $\lim _{z \rightarrow 0+} q_{k}(z)=$ $-\infty$ and $\lim _{z \rightarrow+\infty} q_{k}(z)=-\infty$. Obviously, the points $z_{k, n}$ are the abscissas of the intersection points of the function $q_{k}(z)$ and the brunches of the function $\operatorname{tg} \frac{z}{2}$. By increasing $n$ and $k$, the point $z_{k, n}$ will approach to the point $(2 n-1) \pi$, i.e., $z_{k, n} \sim(2 n-1) \pi$. Hence, from the equality $\sqrt{\lambda-\mu_{k}}=z$, for the eigenvalues of the boundary value problem (2.1), (2.2), satisfying condition $\lambda>\mu_{k}$, we get the asymptotic formula: $\lambda_{k, n}^{(2)} \sim \mu_{k}+(2 n-1)^{2} \pi^{2}$.

Studying equation (3.6), as equation (3.5), we can easily show that in this case the problem $(2.1),(2.2)$ has also two series of eigenvalues, one of which converges to zero and the second one behaves as $\lambda_{k, n}^{(3)} \sim \mu_{k}+(2 n)^{2} \pi^{2}$. Theorem 3.1 is proved.

Remark. The equation similar to equation (3.6) was studied in [2].

Acknowledgements. The author is grateful to the reviewer for many useful comments and corrections, which improved the original manuscript. This work was supported by the Science Development Foundation under the President of the Republic of Azerbaijan -Grant No EIF / MQM/ Elm-Tehsil -1-2016-1(26)$71 / 10 / 1$.

\section{References}

[1] B.A. Aliev, Asymptotic behavior of the eigenvalues of a boundary value problem for a second order elliptic differential-operator equation. Ukr. Mat.Journal 58(8), (2006), 1298-1306. 
[2] B.A. Aliev, Asymptotic behavior of eigenvalues of a boundary value problem for a second order elliptic differential- operator equation with spectral parameter, quadratically occurring in the boundary condition. Diff. Equations, 54(9), (2018), 1256-1260.

[3] B.A. Aliev, N.K.Kurbanova, Asymptotic behavior of eigenvalues of a boundary value problem for a second order elliptic differential-operator equation. Proceedings of the Institute of Mathematics and Mechanics, National Academy of Sciences of Azerbaijan, Special Issue, 40, (2014), 23-29.

[4] B.A. Aliev, N.K. Kurbanova, Ya. Yakubov, Solvability of the abstract Regge boundary value problem and asymptotic behavior of eigenvalues of one abstract spektral problem. Rivista di Matematica della Universita di Parma. 6 (2015), 241-265.

[5] B.A. Aliev, N.K. Kurbanova and Ya. Yakubov, One boundary value problem for elliptic differential-operator equations of the second order with quadratic spectral parameter. Ukrainian Mathematical Journal , 69(6), (2017), 857-875.

[6] B.A. Aliev, N.K. Kurbanova and Ya. Yakubov, Solubility of a boundary value problem for second-order elliptic differential operator equations with a spectral parameter in the equation and in the boundary conditions. Differential equations, 54(1), (2018), 67-85.

[7] B.A. Aliev, Solvability of boundary value problems for fourth order differentialoperator equation with a parameter. Proceedings of the Institute of Mathematics and Mechanics, National Academy of Sciences of Azerbaijan, 43(2), (2017), 261269.

[8] V.I. Gorbachuk, M.A.Rybak, On the boundary-value problems for the SturmLiouville operator equation with a spectral parameter in the equation and in the boundary condition. Direct and inverse problems of the scattering theory. Kiev, (1981), 3-16.(Russian)

[9] N. Yu. Kapustin, On a spectral problem in the theory of the heat operator. Diff. Equations. 45(10) (2009), 1509-1511.

[10] N. Yu. Kapustin, On the uniform convergence in $C^{1}$ of Fourier series for a spectral problem with squared spectral parameter in a boundary condition. Differ. Equations, 47(10), (2011), 1408-1413.

[11] N.B. Kerimov, Kh.R. Mamedov, On a boundary value problem with a spectral parameter in the boundary conditions. Sibir.Mat.Zhurnal 40(2), (1999), 281-290.

[12] M.A. Rybak, Asymptotic distribution of the eigenvalues of some boundary value problems for Sturm-Liouville operator equations. Ukr. Math.J. 32(2), (1980), 159162.

[13] S.Ya. Yakubov, A boundary value problem for the Laplace equation with nonclassical spectral asymptotics. Dokl. AN SSSR, 265(6),(1982), 1330-1333. (Russian)

[14] Ya. Yakubov, Two-fold completeness of root vectors of a system of quadratic pencils. J.Math. Pures Appl. 84 (2005), 1427-1454.

Bahram A. Aliev

Institute of Mathematics and Mechanics of NAS of Azerbaijan, Baku, Azerbaijan, Azerbaijan State Pedagogical University, Baku, Azerbaijan

E-mail address: aliyevbakhram@yandex.ru

Received: December 17, 2018; Revised: July 10, 2019; Accepted: July 17, 2019 